\title{
FROM GRID COMPUTING TO CLOUD INFRASTRUCTURES
}

\author{
Logica Bănică, Cristian Ştefan
}

Faculty of Economics, University of Pitesti, Târgu din Vale, 1, Pitesti, Romania olga.banica@upit.ro

Faculty of Mathematics - Informatics, University of Pitesti, Târgu din Vale, 1, Pitesti, Romania

\section{ABSTRACT}

Icstefan@yahoo.com

As a consequence of the economic crisis, the funds allocated for the development of the IT\&C infrastructure in all domains become scarcer, whilst the need for computing services increases day by day and becomes a key factor in the existence and success of any institution or company.

In the later years, new computing technologies have reached the market, technologies that allow transparent sharing of the physical resources and of the services for their users, like Grid computing paradigms, and lately Cloud infrastructures. In this paper we will conduct a comparative analysis of the architectures and services offered by the two kinds of technologies, aiming to determine if they can give a real competitive advantage to the academic environments through their cost/performance benefits

\section{Indexing terms/Keywords}

Grid computing; Cloud computing; Academic environment; E-learning.

\section{Academic Discipline And Sub-Disciplines}

Information technology; Education;

\section{SUBJECT CLASSIFICATION}

Computer Science

\section{TYPE (METHOD/APPROACH)}

Quasi-Experimental; Comparative Analysis; Designing a virtual academic architecture

\section{Council for Innovative Research}

Peer Review Research Publishing System

\section{Journal: INTERNATIONAL JOURNAL OF COMPUTERS \& TECHNOLOGY}

\author{
Vol 12, No.1 \\ editor@cirworld.com
}

www.cirworld.com, member.cirworld.com 


\section{INTRODUCTION}

Grids and Cloud computing are relatively new computing technologies, that allow transparent sharing of the physical resources and of the services for their users, but also they differ in many respects.

In this paper we will conduct a comparative analysis of the architectures and services offered by the two kinds of technologies, aiming to determine if they can give a real competitive advantage to the academic environments through their cost/performance benefits. In order to build a Grid or Cloud system for the academic environment, we will briefly define the two concepts, their architecture and proposals for typical university implementations.

\section{CLOUD COMPUTING OR GRID COMPUTING?}

Both technologies start from the same vision: to use the computing power of a service provider or a virtual organization, to obtain flexibility, security, storage and platforms and, in the same time, to reduce the cost.

Before presenting the comparative analysis between the two technologies, we will make an overview of the architecture and services they offer.

\subsection{What is Grid computing?}

In 2004, Joseph \& Fellenstein [1] propose an interesting and visionary definition: „In today's pervasive world of needing information anytime and anywhere, the explosive Grid Computing environments have now proven to be so significant that they are often referred to as being the world's single and most powerful computer solutions."

In a nutshell, Grid computing wishes to be a technology which could realize an open collaboration among an infinite number of computing devices connected by open standard protocols, in order to share computing resources in a highly secure manner.

This concept has evolved from 2001, when it was defined by Foster \& Kesselman [2] as „a hardware and software infrastructure that provides dependable, consistent, pervasive, and inexpensive access to high-end computational capabilities". The strength and the important role played by the Grid Computing consists of their capability to manage the IT requirements needed for virtual organizations, meaning that the hardware and software applications provided by a group member will be available to all authenticated users within the grid system.

Actually, Grid computing is more than an aggregate of cooperating resources, it may be considered a new infrastructure, based on web 2.0 access and most of current trends refers to the services-based architectures for grid environments applied in academic, research, industry and business fields.

Business world discovered from the beginning the opportunity to use this infrastructure for business area development. So, IBM developed the Business On Demand system that have „the capacity to sense and respond to fluctuating market conditions in real-time, while providing products and services to customers in an on-demand operational model". [1] In order to solve a problem, a virtual organization based on Grid Computing environment must go through following stages:

the identification of the problem needed to be solved;

the dynamic allocation of the resources from all involved providers;

the provisioning and management of secure access methods to the resources;

the capability to achieve feedback regarding resource status, and process this information to update the application deployment.

\subsection{The architecture of Grid Computing}

The Grid architecture includes five layers, represented in Figure 1: Fabric, Connectivity, Resource, Collective and Application. A suite of standard protocols and services are defined at each level, but the common idea for such a system is its open character.

- The Fabric Layer provides the resources of the Grid: computer clusters and supercomputers, operating systems, mass storage and network devices.

- The Connectivity Layer specifies the protocols for secure Grid-specific network transactions.

- The Resource Layer provides access and management protocols for sharing individual resources and it builds on connectivity layer communication.

- The Collective Layer refers to the collection of Grid resources, allowing to be shared by global protocols and services: Directory brokering, diagnostics, monitoring.

- The Application Layer includes end-user applications based on protocols, standard application programming interfaces (APIs), and Software Development Kits (SDKs) developed in virtual organization [2]. This new infrastructure allows distributing collaborative work to accomplish their tasks that form any complex application.

The integrated Grid Computing Infrastructure provides also Grid Portals, which address various grid middleware issues and it supports applications in the area of Scientific and Engineering, Collaborative Computing, web-enabled applications with problem solving environment as per application requirements [3] 


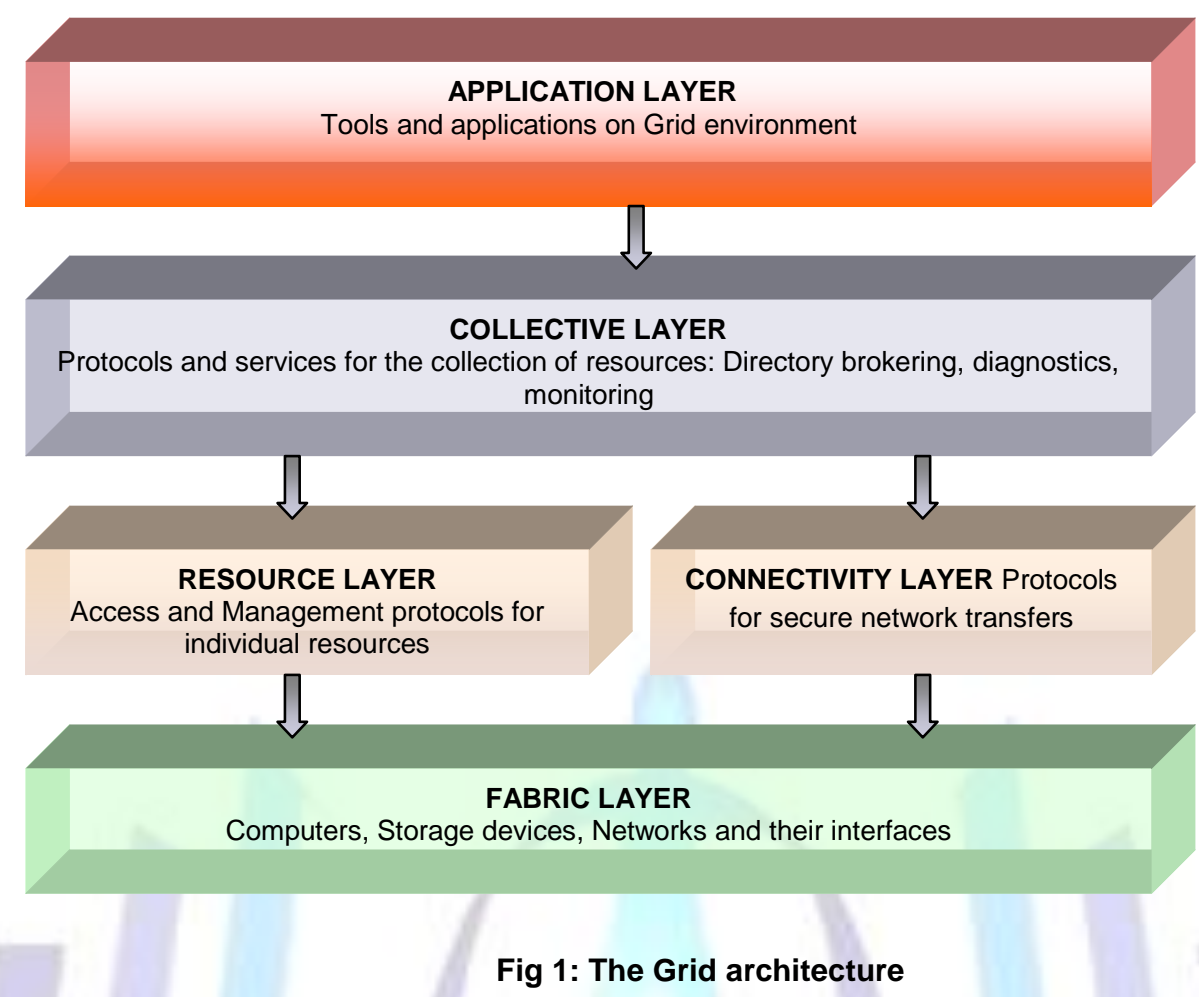

\subsection{Grid computing classification}

There are many types of Grids in the literature, but two of them are more important for the domain: the type of solutions they provide and the size of the organizations involved.

The classification by solutions includes four types of Grids [4]:

a. Computational Grids - are offering computational resources (CPU and GPU) to the research projects that need distributed processing (like medicine, weather and nuclear simulations).

b. Data Grids - are storage-oriented solutions that offer a scalable capacity and reliable data protection for their applications. Information is transparently synchronized between nodes and disk access is accelerated by using the fastest available interfaces, well suited for huge collections of data.

c. Service Grids - reunite hardware resources with the flexibility of hosted operating systems that allow user applications to run and communicate seamlessly. These form a protected environment that clients can rent on a pay-as-you-grow basis.

d. Intelligent Grids - are complete environments that offer all the required levels and include self-management and advanced reporting capabilities that are available remotely. This is the most convenient solution for the unexperienced customers that require high-performance processing.

The classification by size includes three types of Grids:

- Cluster Grid

- Enterprise Grid

- Global Grid

a. Cluster Grid - is a collection of interconnected connected, working as an unique resource at department/group level [4]. Also, Cluster Grid means a suite of computers within the boundary of a Local Area Network (LAN) that offer a single point of access to the organization users.

b. Enterprise Grid - extents the collaboration among different types of resources at the virtual organization level. The Enterprise Grid involves also a number of management politics of resources.

c. Global Grid - is a collection of Cluster and Enterprise Grids, established over the Internet to provide individuals or organizations with grid power. This solution is based on general sharing protocols of the resources and a global politic of resource use.

There were developed large-scale infrastructure for open scientific research, as TeraGrid (developed by the National Science Foundation's - NSF), Open Science Grid, Earth System Grid, European Grid Infrastructures, etc.

Researchers, engineers, students, and other scholars may obtain free access to high performance computing, after submitting a proposal outlining for their in order to obtain an initial allocation. 


\subsection{What is Cloud Computing?}

Cloud Computing is another manner to access computing resources and web 2.0 based- services, the most common implementation being the one in which an external partner is the owner of the infrastructure and it provides cloud services pay-per-use. It is a method to reduce the costs with hardware and software platforms, and to increase the IT performances of a small and medium firm in a high-level of security environment.

Grid Computing enables the users to share and benefit from computational resources in a virtual organization, whilst computing Clouds enable the use of IT services without having any infrastructure, only a credit card to rent the resources from powerful software companies as Google, Microsoft, Amazon, etc.

Before making an analysis by comparing the two technologies, a brief description of the Cloud Computing concept is given.

In literature, Cloud computing is defined as "a model for enabling convenient, on-demand network access to a shared pool of configurable computing resources (networks, servers, storage, applications, and services) that can be rapidly provisioned and released with minimal management effort or service provider interaction" (Mell \& Grance, 2011 - [5]).

The main features of cloud computing solutions can be summarized as follows (Cacciari et al., 2010 - [6]):

- use of Internet technologies to offer scalable and flexible services; this feature refers to the capacity of dynamically acquiring resources which support variable-size tasks, that can be measured and are cost-effective;

- full maintenance and security are offered by providers; they have more efficiency, extended know-how and the possibilities to periodically update the hardware and software resources.

- data storage strategy takes into account the physical distance to the site where resources are used in a locationindependent manner; this leads to increases in reliability, security and lowers communication costs.

- by dynamically provisioning valuable resources, cloud computing platforms can reassign the unused resources and move them to where consumer demand is the highest.

A characteristic of cloud computing is the possibility to be accessed anywhere from an Internet connection and to promote on-demand self-service, by helping the end users to have computing resources at their disposal without the need of permanent interaction with the service provider. Data may be accessed from any network, regardless of the client platform that is used.

Cloud computing can be implemented according to three fundamental models (Gray, 2010 - [7], O’Neill, 2009 - [8], Williams, $2009-[9])$ :

- $\quad$ Infrastructure as a Service (laaS) - the capability provided to the user to provision processing, storage, networks, and other computing resources as operating systems and encapsulated resources as virtual clusters

- Platform as a Service (PaaS) - the capability provided to the user to build, test and deploy consumer-created or acquired applications onto the cloud infrastructure. This layer offers a collection of specialized tools, middleware and services designated to provide a development platform. The developers don't have any control to the cloud infrastructure, but they have control over the deployed applications and possibly application hosting environment configurations.

Software as a Service (SaaS) - provides business application in the cloud; in this model cloud providers install and operate application software on their platform and users access the services remotely. The cloud users do not manage the cloud infrastructure on which their application is running. The application is not installed and does not run on client computers; in this manner the customer is not responsible for maintenance and support for the software.

Figure 2 shows a generic architecture of the main domain of social and business domains powered Cloud, as a layered structure.

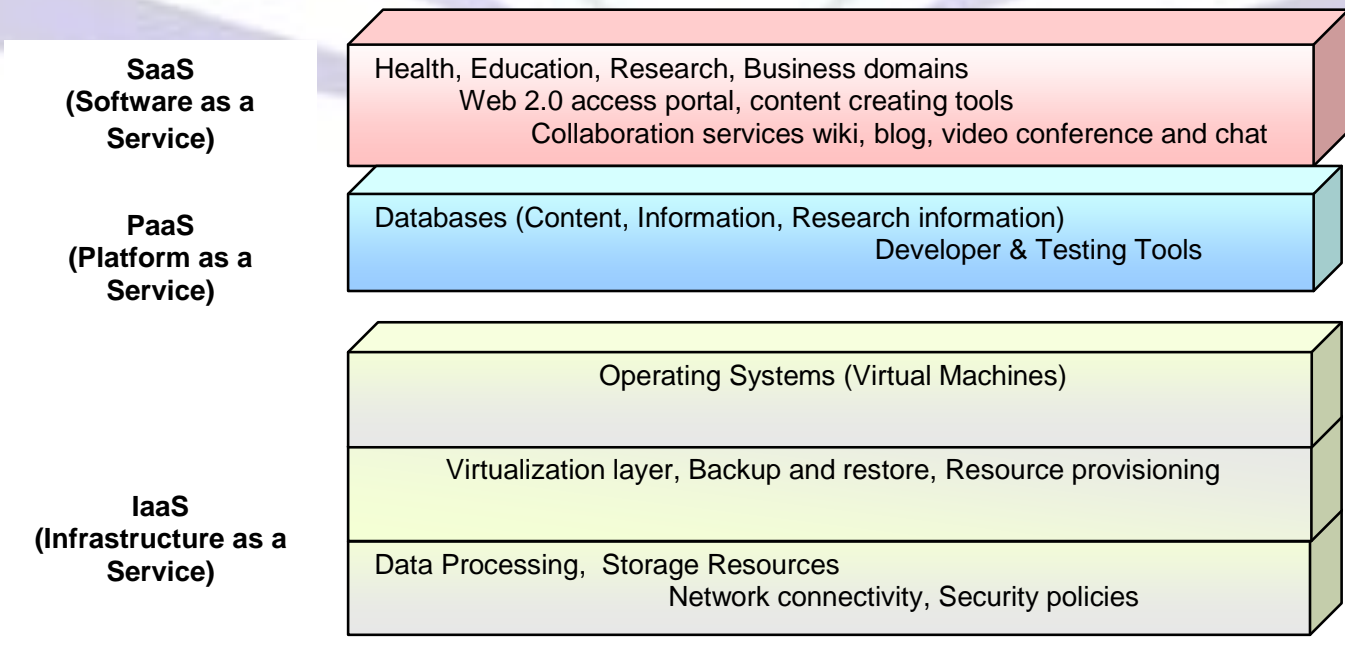

Fig 2: The layered architecture of Cloud Computing 
The main classification of Cloud Computing implementations is by the size and the access level provided:

Public Cloud - the infrastructure is owned by a powerful software company, providing cloud services available to the general public or large groups of users; their services are free or offered on a pay-per-use model.

- $\quad$ Private Cloud - the infrastructure is owned, operated and managed by a private organization for its internal use only;

- Community Cloud - the infrastructure is shared by several organizations and supports a specific community that has similar approaches about policy, objectives, and security requirements.

Hybrid Cloud - the infrastructure is a joint solution of two or more clouds (private, community, or public) that are bound together by standardized rules that enable data and application portability.

\section{THE EVOLUTION FROM GRID COMPUTING TO CLOUD COMPUTING}

In the following part we will present a comparative analysis of the two technologies and finally we will discuss the arguments that allow us to affirm that we have reached an evolution by migrating from grid computing to cloud services.

- The first question that comes to mind is "who are the providers of such systems?"

- for the grid technologies, the involved organizations and institutions are sharing resources and are based on open standards that allow an easy collaboration; resources are widely spread geographically and are connected though open source standards, managed by the providers;

- an infrastructure that spans across multiple virtual organizations (VO) each of them can consist of either physically distributed institutions or logically related projects/groups. The goal of such a paradigm is to enable federated resource sharing in dynamic, distributed environments.

- for the Cloud systems, powerful software companies are the ones that offer the resources and IT services; resources are concentrated in the datacenters, and the administration and security aspects are known only to the provider;

- The second question is related to the potential users that are targeted

- organizations and institutions that share resources and that form a virtual organization

- small and medium-sized companies and end-users that need IT services and have broadband access to the Internet; there are no pre-defined use-cases, each client builds his own application and allocates resources on demand.

Each technology has is strengths and drawbacks, and their knowledge opens the way for a complete analysis and a welldefined conclusion.

Which are the advantages of each technology?

- grid systems offer shared resources, transparent access and redundancy, and if a datacenter fails, another one can take the tasks;

- cloud infrastructures offer their users affordable services that are easy to use and deploy

What are the drawbacks?

- $\quad$ grid computing platforms are complex and cumbersome to integrate with the existing IT infrastructure of the organization without the required knowledge; also, they are built each for a very special purpose

- cloud services don't offer high-level specialized services, like the Grids, are not transparent and are concentrated in a single place, thus prone to natural disasters

The above arguments lead to the conclusion that the Cloud technologies and Grid systems are based on the same concept: common access to a powerful infrastructure, which the users can take advantage of without really owning it.

The evolution starts from the focus on shared computing and storage resources (like Grids) and reaches the public services offer by the Cloud.

In general, the whole range of service-oriented applications is based on the Web 2.0 and this offers these technologies a high impact on the academic environment, and also for the health, advanced research and online business sectors.

Grid Computing aims to "enable resource sharing and coordinated problem solving in dynamic, multi-institutional virtual organizations" (Foster et al., 2001 - [10]).

The vision for Clouds and Grids are similar, but the technologies used differ, so we tried to find some obvious contrasts between them, regarding the services offered, the providers and the users. Even the Cloud computing services are not yet ready for high-level performance as the Grids offer, the first technology is more and more preferred by the small and medium companies, but also by the home users because its simplicity, its large distribution and the way of payment on consumption basis (Foster, 2008 - [11]).

The business model for the Grids is project-oriented, meaning that the users or community represented by that proposal have certain number of service units (CPU hours) they can spend. For example, the TeraGrid operates in this fashion, and requires increasingly complex proposals to be written for increasing number of computational power. The TeraGrid has more than a dozen Grid sites, all hosted at various institutions around the country [11]. 
Other notable examples of academic research grids include Planet Lab and Emanics Lab (which are now a single entity). They offer their users spread across all European countries access to a distributed computing infrastructure in exchange for two nodes that run the grid software. This allows for complex experiments to take place simultaneously.

Finally, after this close comparison, we consider that Cloud Computing is an evolving infrastructure of Grids and, in the future, its actual implementations will became large-scale systems.

\section{USING THE TWO TECHNOLOGIES IN ACADEMIC ENVIRONMENT}

\subsection{A proposal of a Grid system for a university}

In the academic environment, Grids can be built on many layers, with an increasingly higher degree of complexity:

- inside an university, by sharing only the private resources;

- $\quad$ within a consortium of universities, by sharing the resources of the involved institutions;

- $\quad$ nation-wide, by implementing a governmental project that reunites and makes available all shareable resources from the country's universities to the academic world.

In this paper, we are interested to demonstrate the advantages of grid computing systems over isolated computing resources (servers and other distributed systems) at university level.

There is a negotiation of resources sharing arrangements among the user entities in order to execute the tasks in the most efficient manner (Al-iesawi \& Samat, 2010, 2010 - [12]).

The distributed resources of the university are organized in local area networks - Cluster Grids, interconnected in Enterprise Grids that host all the servers and storage resources that are involved in the Grid structure, and must be connected together as a single internal network, accessible as a public network.

The next level aims to interconnect universities from within the country that are willing to collaborate and share resources, and especially information.

At national level, all the universities, coordinated by the national institutions and ministries, and other statutory bodies cooperate in order to become the part of a single academic grid system.

The university Grids must have a minimum number of servers, which should accomplish the main tasks for the architecture:

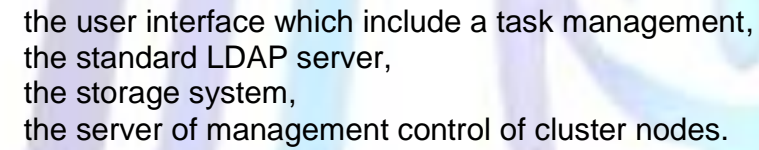

Each grid server requires a public Internet Protocol (IP) address and also, if the WNs need to be accessed directly by servers from other connected grid systems, they also need public IP addresses (Qiang \& Konstantinov, 2010 - [13]).

Once the hardware of the grid system and the network have been installed, the appropriate software layers can be deployed, especially the operating systems, middleware and virtualization software. It is recommended to choose free open source software in order to minimize the cost of the implementation, like Linux family as operating system, MySQL as database server and Proxmox 1.8 as virtualization platform.

To best use the resources available and respond to the requirements of the Grid at their best, a number of design decisions were made, based on the needs of the academic environment. Firstly, because the workstations could arbitrarily be running in either Linux or Windows at any time, both operating systems needed to be configured as grid nodes as similarly and as simply as possible. The highly dynamic nature of these workstations led to the first design decision using the P2P infrastructure of ProActive. This is a peer-to-peer infrastructure completely decentralized and consequently, the grid would not have to rely on any one node to function. It also allows the workstations to join and leave the Grid as users require or restart their operating systems.

Of particular interest is developing Web Services that provide an interface to the Grid system. The Globus Toolkit is a widely-used infrastructure for Grid computing [15], and would form the basis of an excellent comparison to this research.

A Globus-based grid could be deployed and a similar application could be developed for testing and benchmarking against that presented in this paper.

\subsection{Cloud computing for a university}

Starting from the main difference between the two technologies, defined by the fact that Cloud infrastructures are based on public access to centralized resources, we have discussed in a previous paper the importance of Cloud Computing for the academic environment and we've highlighted some major characteristics for e-learning on such an implementation.

A scenario for Cloud-based e-Learning architecture involves the following important stages: 
- A powerful infrastructure should be available, including all the necessary hardware and software computing resources involved in e-learning activities;

- A unifying solution for the Learning Objects representation, taking in account that the vast majority of the universities have their own Learning Management System, implemented in private clouds, but it is a different one for each institution;

- The access of students and teachers of the universities involved in the e-Learning Cloud project to all available educational content and to the collaboration tools;

- The security and the confidentiality of scholar records, teaching personnel data and research results.

In Figure 3 (Banica et al., 2013 - [14]) is shown the e-Learning Cloud architecture proposed for the Romanian universities.

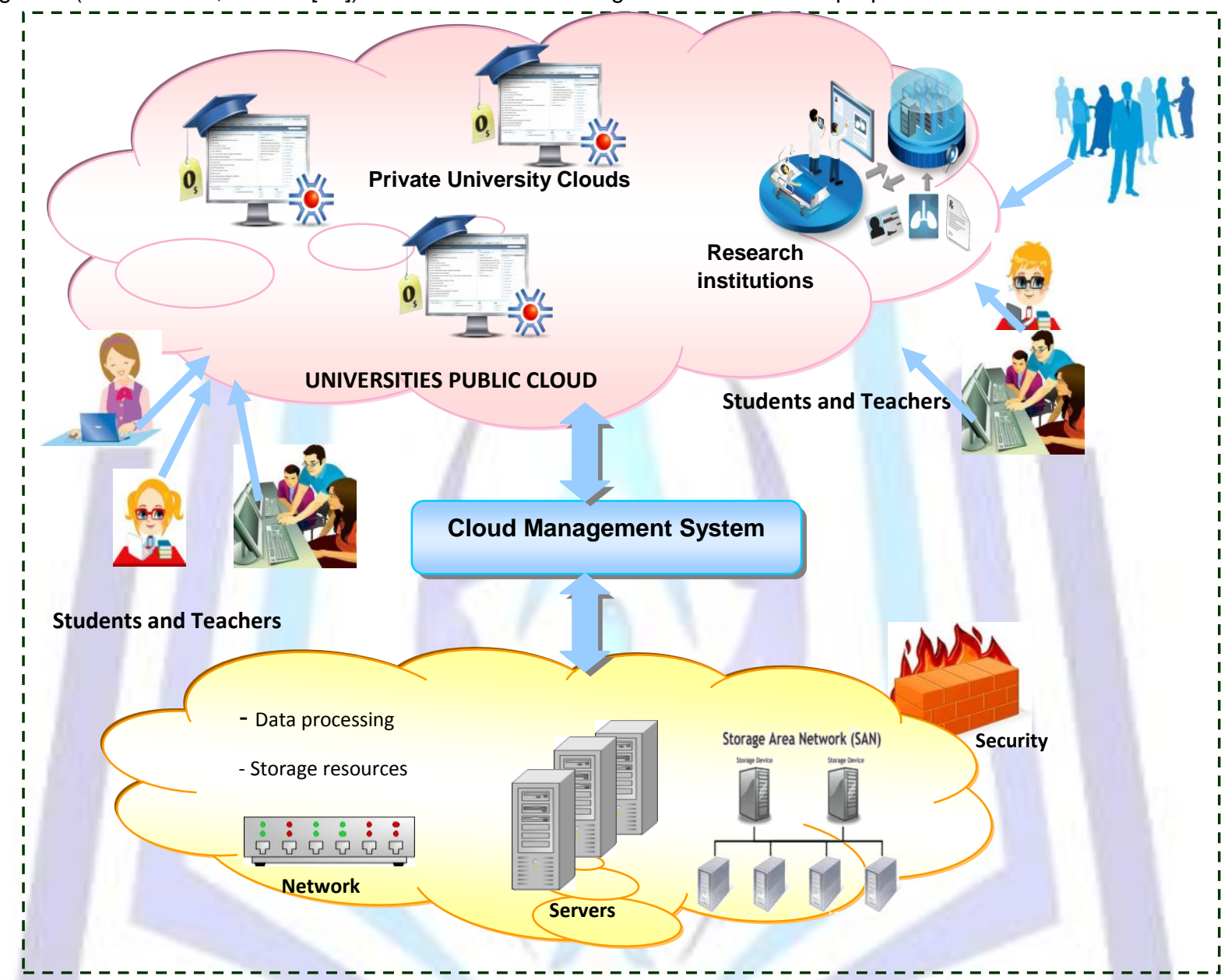

Fig 3: E-Learning Cloud architecture proposed for the Romanian universities

Source: Banica et al., 2013 - [14]

In order to implement a Cloud Management System, there are two options available, one based on the proprietary IBM technology (IBM SmartCloud), and the other based on the open-source cloud-hosted Docebo solution.

Obviously, they differ both in qualitative and pricing aspects, though we considered that Docebo can be a reasonable comparison when developing an e-Learning Cloud and testing the solution.

The migration to the IBM SmartCloud solution involves important financial resources that only a consortium of multiple superior education institutions could support, as it will be too expensive for a single university to develop. IBM SmartCloud includes infrastructure as a service, software as a service and platform as a service offered through public, private and hybrid cloud delivery models. IBM also builds cloud environments for clients that are not necessarily on the SmartCloud Platform.

The second proposal for a Cloud e-Learning in academic environment is based on Docebo Cloud Learning Management System that is a Saas e-Learning platform and doesn't require any download or installation. It can be activated online, from Docebo's website [15].

There is also a free alternative that iTeach open to any person interested in offering their students a hosting platform for their educational content, involving the Claroline or Moodle LMSs and 2GB of e-mail storage. This option is completely free, with no trial period, and is worth mentioning although the space for content is limited to $100 \mathrm{MB} /$ teacher account. 


\section{CONCLUSIONS}

In order to build a Grid or Cloud system in an academic environment, it is necessary to have a full vision of the future of these two technologies and correct evaluation of internal resources of the university. Also, it is very important to know the tools for managing the underlying resources and the distributed computations.

Comparing Grid systems and Clouds infrastructures side-by-side from a future perspective we believe that the competition will be between a common interface solution to grid services (Open Grid Service Architecture) and the commercial Cloud services, rather than with a specialized system built for the specific internal environment.

\section{REFERENCES}

[1] Joseph, J., Fellenstein, C., 2004. Introduction to Grid Computing, available at http://vig.prenhall.com/catalog/academic/product/0,1144,0131456601,00.html

[2] Foster, I., Kesselman, C. and Tuecke, S. (2001) International Journal of High Performance Computating Applications 15(3), 200, available at http://www.globus.org/research/papers/anatomy.pdf.

[3] Nath, K., 2013. A Review on Grid Computing, International Journal of Information and Computation Technology, ISSN 0974-2239 Volume 3, Number 5 (2013), pp. 445-450, available at http://www. irphouse.com /ijict.htm

[4] Kurdi, H., Li, M., Al-Raweshidy, H., 2008. A Classification of Emerging and Traditional Grid Systems, IEEE Computer Society, Vol. 9, No. 3, art. no. 0803-mds2008030001 1541-4922, available at http://www.computer.org/csdl/mags/ds/2008/03/mds2008030001-abs.html

[5] Mell, P., Grance, T., 2011. National Institute of Standards and Technology - Definition of Cloud Computing, available at http://csrc.nist.gov/groups/SNS/cloudcomputing/index.html

[6] Cacciari, C., D’Andria, F., Gonzalo, M., Hagemeier, B. et al., 2010. ElasticLM: A novel approach for software licensing in distributed computing infrastructures.", Procedures IEEE 2nd International Conference on Cloud Computing Technology and Science, pp. 67-74.

[7] Gray, M., 2010. Cloud Computing: Demystifying laaS, PaaS and SaaS., available at http://www.zdnet.com/news/cloud-computingdemystifying-iaas-paas-and-saas/477238.

[8] O'Neill., M., 2009. SaaS, PaaS, and laaS: A security checklist for cloud models, available at: http://www.csoonline.com/article/660065/saas-paas-and-iaas-a-securitychecklist-for-cloud-models.

[9] Williams, A., 2009. The Feds, not Forrester, Are Developing Better Definitions for Cloud Computing, available at http://www.readwriteweb.com/enterprise/2009/10/forrrester-says-we-need-better.php.

[10] Foster, I., Kesselman, C., Tuecke, S, 2001. The anatomy of the Grid: Enabling scalable virtual organization. The International Journal of High Performance Computing Applications, 15(3), pp. 200--222.

[11] Foster, I., Zhao, Y., Raicu, I., Lu, S., 2008. Cloud Computing and Grid Computing 360-Degree Compared, Grid Computing Environments Workshop, pp. 1-10.

[12] Al-iesawi, A. M., Samat, M. I. M., 2010. A case study on implementation of grid computing to academic institution, International Symposium in Information Technology, pp.1525 - 1530

[13] Qiang, W., Konstantinov, A., 2010. The design and implementation of standards-based grid single sign-on using federated identity, 12th IEEE International Conference on High Performance, Computing and Communications, pp. 458 - 464

[14] Banica L., Stefan C., Rosca D. \& Enescu F., 2013. Moving from learning management systems to the e-learning cloud, AWERProcedia Information Technology \& Computer Science. [Online], pp 865-874, available from: www.awer-center.org/pitcs

[15] http://www.docebo.com/saas/index.php?r=site/index\&lang=en [Accessed 2th December 2013]

[16] Banica, L., Pirvu, D., Stefan, C., 2012. Distributed Business Intelligence - A New Approach In Economical Education, International Journal of Computers \& Technology, [Online] 2012, 3(3), pp 413-418, available from: www.ijctonline.com 